\title{
Analysis of econometric research hotspots and application prospects _ visualization research based on citespace
}

\author{
Xiaolin Sun ${ }^{1}$, Menghe Zhao ${ }^{1, *}$ \\ ${ }^{1}$ Department of Management, Dalian Polytechnic University, China
}

\begin{abstract}
By analyzing scholars' research on econometrics, we can understand the hot topics of related research and, based on the changes of research hotspots in different years, we can derive the future application prospects. In this paper, the literature related to econometrics in cnki and web of science is selected as the sample source, and the literature between 2010 and 2020 is analyzed by the visualization software citespace for authors, keywords, etc. Through the study, it is found that the application of spatial econometrics and the solution to practical problems are the focus of current scholars' research, and the application of econometrics is very wide, and the solution to practical problems is the new trend of econometric research in the future.
\end{abstract}

\section{Introduction}

According to Frisch, econometrics is the combination of statistics, economic theory and mathematics, using mathematics, statistical methods and computer technology to quantitatively analyze and study the relationship between economic variables with stochastic characteristics, using econometric models as the main means to accurately and efficiently describe economic phenomena.

There are three main applications of econometrics: microeconometrics, macroeconometrics and financial econometrics. The application of microeconometrics is to analyze economic problems in the areas of household finance, labor and housing, and business behavior, reveal the problems, and put forward corresponding countermeasures and suggestions; the application of macroeconometrics is to study economic fluctuations, fiscal and tax policies, and trade exchange rates; and the application of financial econometrics is mainly focused on asset pricing, corporate finance, and The application of financial econometrics is mainly focused on asset pricing, corporate finance and Internet finance to provide targeted suggestions for maintaining the stability of financial markets ${ }^{[1]}$. With the development of computer technology, the methods and accuracy of econometrics have been improving, and many scholars have studied the shortcomings and limitations of econometrics and proposed solutions to ensure the credibility of research and explore the application prospects of econometrics ${ }^{[2-4]}$.
In this paper, we will analyze the current research hotspots and the future application prospects through citespace to collect statistics of the literature about econometrics at home and abroad in the past ten years.

\section{Data sources and methods}

The foreign literature data were retrieved from Web of Science with "title = Econometrics" as the search condition, and the search range was 2010-2020. 3895 articles were retrieved. The data of Chinese literature were retrieved from CNKI with "title=econometrics" as the search condition, and the search range was 20102020 , and a total of 2703 relevant papers were retrieved.

In this paper, we use the citespace software developed by Prof. Chaomei Chen to visualize and analyze the literature, so as to visualize the current research hotspots of econometrics and the future application prospects.

\section{Analysis of domestic and international econometric studies}

\subsection{Analysis of research scholars}

The authors of Chinese literature and the co-cited authors of English literature are analyzed by citespace, and the scholars with higher volume of articles and higher frequency of citations are obtained respectively, 


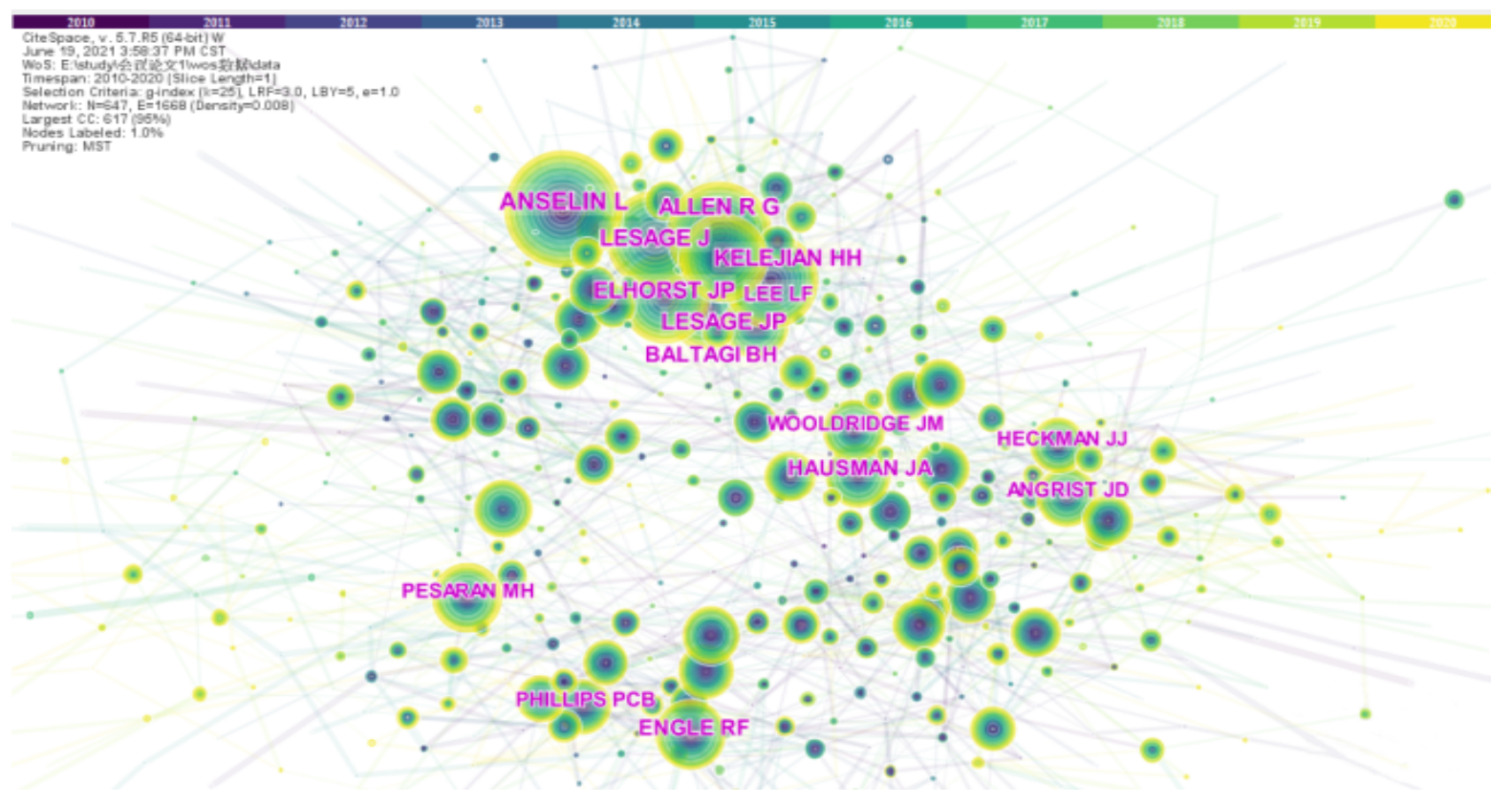

Fig. 1. Cited author of Foreign Econometric Studies

so as to analyze the more authoritative scholars in this field, and then analyze their academic views and works, and derive the hot spots of general interest in the field of econometrics. The co-cited knowledge graph is shown in Fig. 1, and the statistics of scholars' publications are shown in Table 1.

Through Fig. 1, it is found that the most frequently cited foreign scholar from 2010 to 2020 is ANSELIN L, with 515 citations. ANSELIN L's main research direction is spatial econometrics, which has made a great contribution to making spatial econometrics a mainstream econometrics and making the application field of spatial econometrics more extensive. Spatial Econometrics: Methods and Models is the best known work of ANSELIN L and has provided an important basis for the research of other scholars. Other scholars such as ALLEN R G and LASAGE J are also cited more frequently, providing more accurate research methods and models for econometrics.

Table 1. Number of articles by domestic authors

\begin{tabular}{cc}
\hline Author & Posting volume \\
\hline Liyan Liu & 8 \\
Ming Liu & 6 \\
Min Zhang & 5 \\
Zinai Li & 5 \\
Kunyang Wan & 5 \\
Mao Zhang & 5 \\
Jinxia Wang & 5 \\
\hline
\end{tabular}

Through Table 1, it is found that the highest number of articles among China research scholars from 2010 to 2020 is Liyan Liu, with 8 articles, and her main research direction is the theory of econometrics and its limitations ${ }^{[5-7]}$, which has contributed to improving the accuracy of econometric research. Other scholars focus on the application of models ${ }^{[8-10]}$, teaching reform ${ }^{[11]}$ and practical problem analysis and solution ${ }^{[12-14]}$.

An analysis of the literature from 2010-2020 shows that scholars are concerned with econometrics, and scholars have made extensive research on the basic theory and practical applications of econometrics.

\subsection{Analysis of research hotspots}

The knowledge map of research hotspots is shown in Figure 2, where the color and thickness of the annual wheel indicate the time and number of keywords respectively, and the node size indicates the frequency of keywords.

As can be seen from Figure 2, the high-frequency keywords of foreign studies focus on "econometrics", "model", "spatial econometrics", "specification", "regression", "panel data", and "spatial econometrics". The key words "panel data" and "economic growth" indicate that the hot spots of foreign research in recent years have focused on the field of spatial econometrics and the analysis of models and data. 


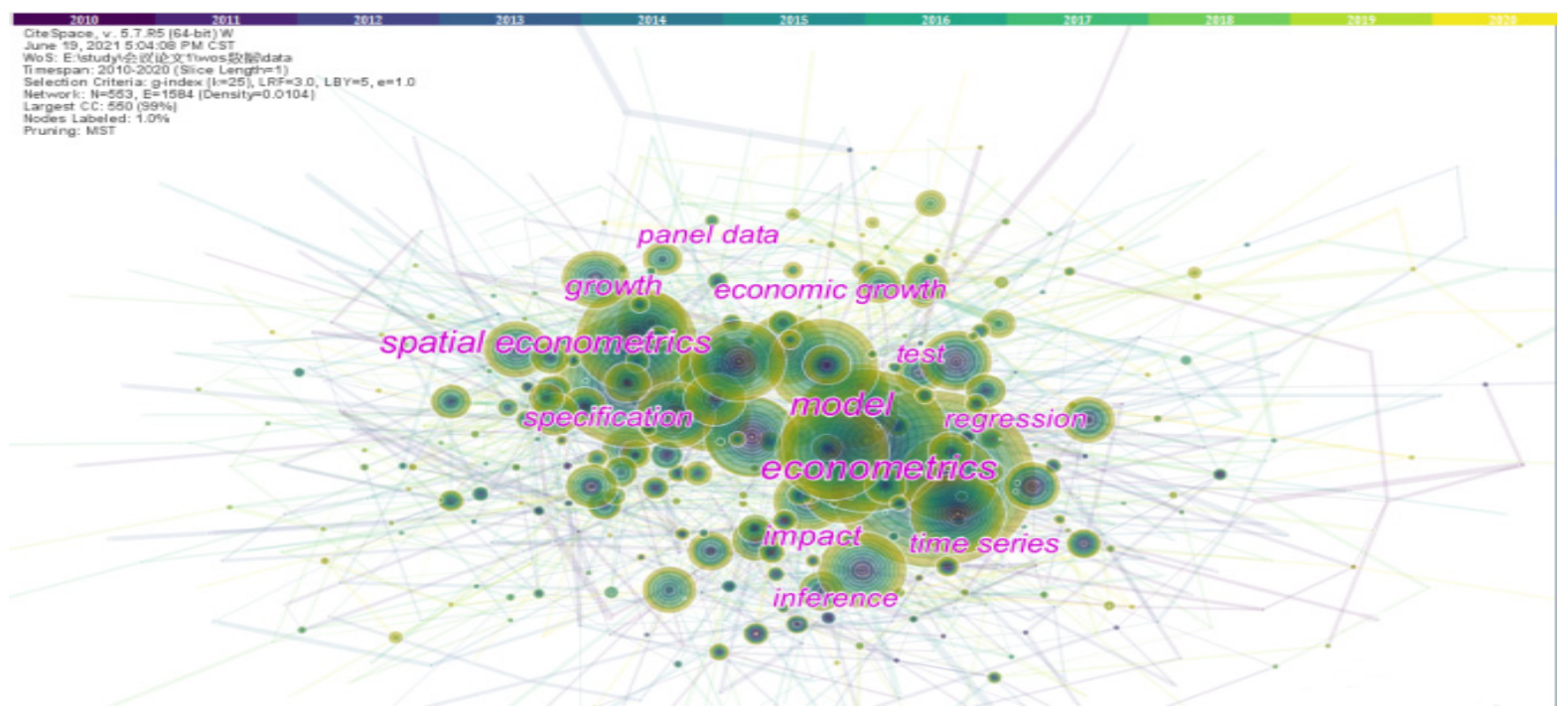

Fig. 2. Knowledge mapping of foreign econometric research hotspots

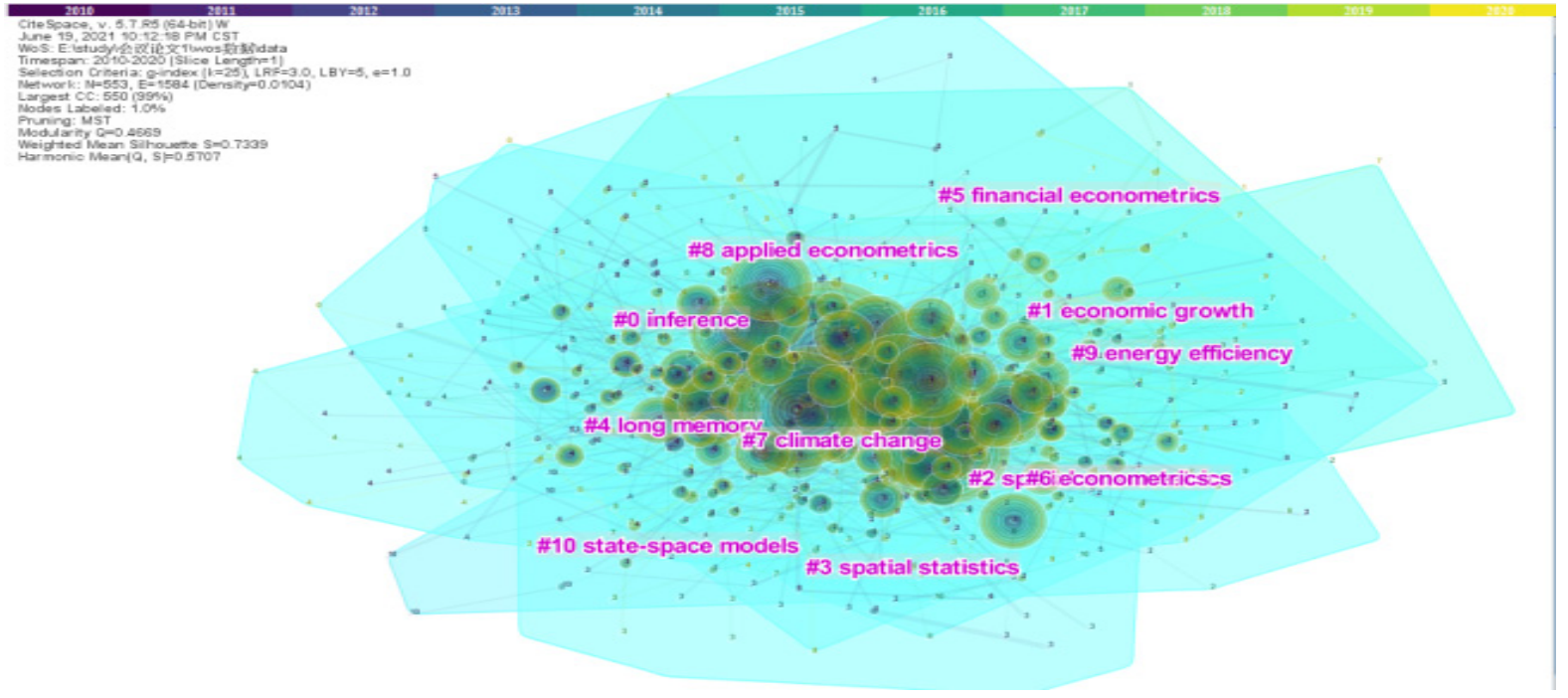

Fig. 3. Clustered view of foreign econometric research hotspot

The main clusters are "inference", "economic growth", "spatial econometrics", "long memory", and "financial econometrics". econometrics", "long memory", "financial econometrics ", "econometrics", "climate change", "applied econometrics", "applied econometrics", "energy efficiency", thus it can be seen that foreign research is biased towards the application of econometrics in various fields, including environment, energy, artificial intelligence and many other In this way, it can be seen that the foreign research focuses on the application of econometrics in various fields, including environment, energy, artificial intelligence, etc.

In summary, scholars' research on econometrics in recent years has favored the field of spatial econometrics, mainly because spatial econometrics can analyze spatial data, so the application will be more extensive and the analysis results will be more accurate. In addition, scholars have applied econometric analysis to various fields, which is of great significance to the solution of practical problems.

\subsection{Research Frontier Analysis}

A timeline diagram of the keyword analysis of the literature can demonstrate the changes in research hotspots in recent years, and the resulting timeline diagram is shown in Figure 4, which shows the year in which each keyword appears and the concentration of research topics.

From Figure 4, it can be seen that foreign scholars have been focusing their research on the application of econometrics in the period of 2010-2020 to solve the problems of "climate change", "energy It also focuses on the research of econometrics for economic growth, 


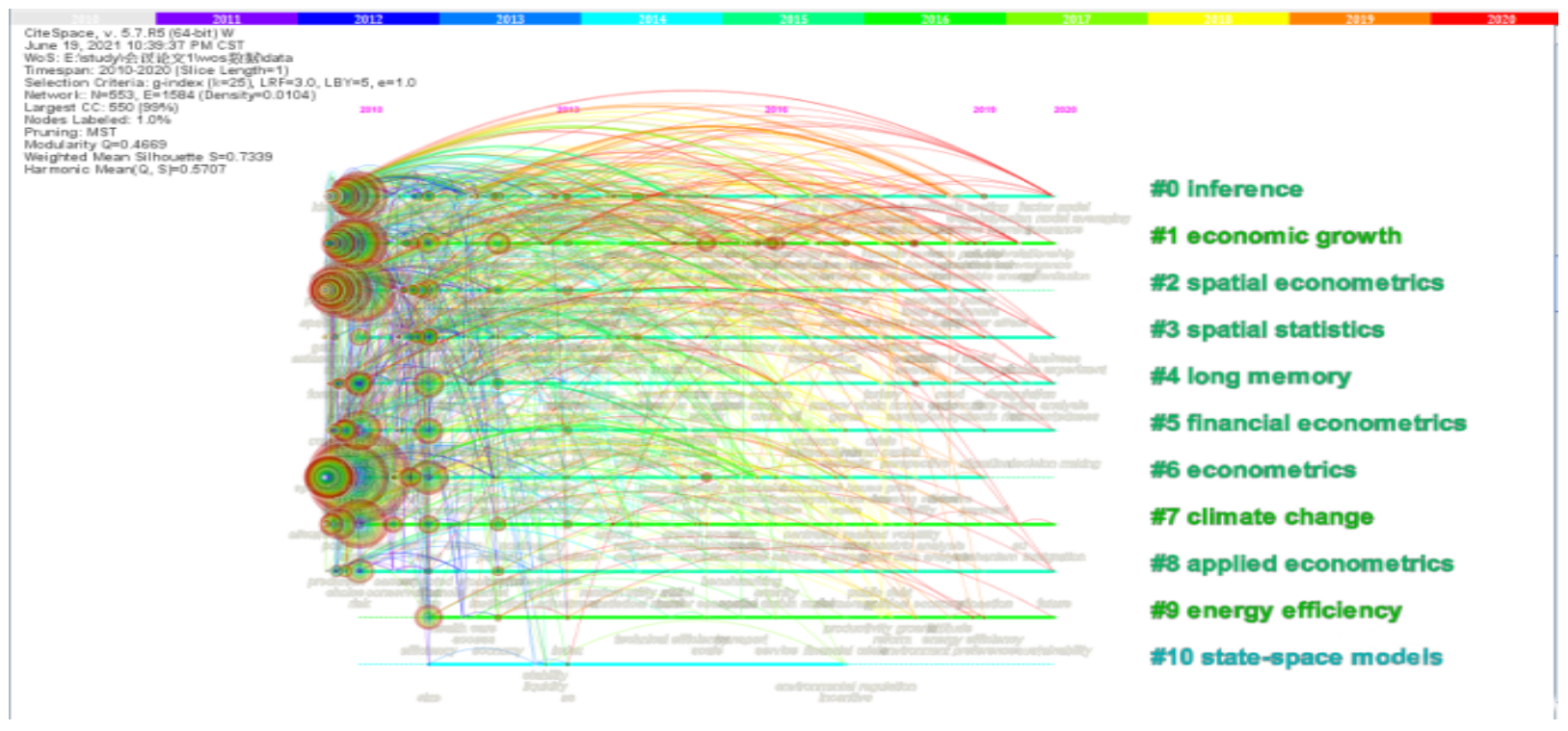

Fig. 4. Knowledge mapping of foreign econometric research frontiers

which is also important for the research of macroeconomic policies.

Through the above analysis, it can be seen that the research of econometric theories and models has been a hot spot for scholars, which can ensure that the research methods and models of econometrics are continuously improved. In addition, spatial econometrics has been widely used due to its ability to analyze data accurately and has a good application prospect. In addition, scholars have also applied econometrics to solve current hot issues, such as environment, energy and artificial intelligence, which indicates that the research trend on econometrics will be biased toward practical applications, which is important for the improvement of policies and measure.

\section{Conclusion}

In this paper, by using citespace to analyze the relevant literature on econometrics from 2010 to 2020, the following conclusions are drawn: first, scholars focus on the research of basic theories and models of econometrics; second, spatial econometrics has a better application prospect because of its unique advantages in the analysis of spatial data; Third, the analysis and solution of practical problems and social hotspots are the new trends of econometric research in the future.

\section{Acknowledgements}

Fund Project 1. 2017 Basic Research Projects of Higher Education Institutions in Liaoning Province "Institutional Environment, Cross-regional M\&A and Industrial Upgrading Effect" (2017J067).

Dalian Social Science Federation Project in 2020, Dalian High Quality Development Level Evaluation and Path Research (Key Project, 2020dlskzd212).
First-class applied majors in Liaoning Province: Business Administration, Liaoning Provincial Department of Education, 2019.

\section{References}

1. $\mathrm{Yi} \mathrm{Hu}, \mathrm{Haiqiang}$ Chen, Yingfei Qi, Economic Research, 54,5(2019).

2. KeYun Zhang, MengYu Yang, Business Review, 7, 7 (2016).

3. Chengquan Zhu, Liyan Liu, The Economist, 8(2013).

4. ZN Li, LSh Qi, Quantitative Economic and Technical Economics Research, 27,14 (2010).

5. Liyan Liu, Research on financial issues,9 (2014).

6. Liyan Liu, Research on financial issues, 12(2013).

7. Liyan Liu, Dongbei University of Finance and Economics, (2012).

8. Ming Liu, Statistics and Decision-making,5 (2016).

9. M Liu,HJ Huang, Statistics \& Information Forum, 29,6 (2014).

10. ZN Li,KP Li, Statistical Research,26,6(2009).

11. Min Zhang, Curriculum Education Research, 2(2019).

12. Huang Chen, Jinxia Wang, Jikun Huang, Journal of Natural Resources, 27,2 (2012).

13. Kunyang Wan, Wencong Lu, Scientific Research,28,10(2010).

14. Mao Zhang, Dongyan Qiao, Yongan Dai. Scientific Decision Making,7 (2010). 